\title{
Oesophageal IGRT considerations for SBRT of LA-NSCLC: barium-enhanced CBCT and interfraction motion
}

\author{
Katrina Woodford ${ }^{1,2^{*}}$, Vanessa Panettieri ${ }^{1,3}$, Jeremy D. Ruben ${ }^{1,2}$, Sidney Davis ${ }^{1,2}$, Trieumy Tran Le ${ }^{1}$, \\ Stephanie Miller ${ }^{1}$ and Sashendra Senthi, ${ }^{1,2}$
}

\begin{abstract}
Background: To determine the optimal volume of barium for oesophageal localisation on cone-beam CT (CBCT) for locally-advanced non-small cell lung cancers (NSCLC) and quantify the interfraction oesophageal movement relative to tumour.

Methods: Twenty NSCLC patients with mediastinal and/or hilar disease receiving radical radiotherapy were recruited. The first five patients received $25 \mathrm{ml}$ of barium prior to their planning CT and alternate CBCTs during treatment. Subsequent five patient cohorts, received $15 \mathrm{ml}, 10 \mathrm{ml}$ and $5 \mathrm{ml}$. Six observers contoured the oesophagus on each of the 107 datasets and consensus contours were created. Overall 642 observer contours were generated and interobserver contouring reproducibility was assessed. The kappa statistic, dice coefficient and Hausdorff Distance (HD) were used to compare barium-enhanced CBCTs and non-enhanced CBCTs. Oesophageal displacement was assessed using the HD between consensus contours of barium-enhanced CBCTs and planning CTs.
\end{abstract}

Results: Interobserver contouring reproducibility was significantly improved in barium-enhanced CBCTs compared to non-contrast CBCTs with minimal difference between barium dose levels. Only $10 \mathrm{~mL}$ produced a significantly higher kappa $(0.814, p=0.008)$ and dice $(0.895, p=0.001)$. The poorer the reproducibility without barium, the greater the improvement barium provided. The median interfraction HD between consensus contours was $4 \mathrm{~mm}$, with $95 \%$ of the oesophageal displacement within $15 \mathrm{~mm}$.

Conclusions: $10 \mathrm{~mL}$ of barium significantly improves oesophageal localisation on CBCT with minimal image artifact. The oesophagus moves substantially and unpredictably over a course of treatment, requiring close daily monitoring in the context of hypofractionation.

Keywords: Oesophagus, Barium, Contrast, IGRT, Interfraction motion, CBCT, SBRT

\section{Background}

Up to $70 \%$ of patients with potentially curable locallyadvanced non-small cell lung cancer (NSCLC) do not receive radical treatment and as many as $36 \%$ receive no treatment at all [1-4]. Many factors play into why

\footnotetext{
*Correspondence: K.Woodford@alfred.org.au

${ }^{1}$ Alfred Health Radiation Oncology, The Alfred, 55 Commercial Road, Melbourne, VIC 3004, Australia
}

Full list of author information is available at the end of the article this might be the case, but concerns regarding competing comorbidities, oesophageal toxicity and the logistic requirements of attending daily treatment for 6 weeks are foremost. With increasing access to stereotactic body radiotherapy (SBRT), there is an ever-increasing proportion of early stage NSCLC patients receiving radical treatment [5-7]. SBRT may have a similar impact if applied in the locally advanced setting. The safety of a hypofractionated regime of $15 \times 4$ Gy-fractions original author(s) and the source, provide a link to the Creative Commons licence, and indicate if changes were made. The images or other third party material in this article are included in the article's Creative Commons licence, unless indicated otherwise in a credit line to the material. If material is not included in the article's Creative Commons licence and your intended use is not permitted by statutory regulation or exceeds the permitted use, you will need to obtain permission directly from the copyright holder. To view a copy of this licence, visit http://creativecommons.org/licenses/by/4.0/. The Creative Commons Public Domain Dedication waiver (http://creativeco mmons.org/publicdomain/zero/1.0/) applies to the data made available in this article, unless otherwise stated in a credit line to the data. 
has been demonstrated [8-10] and is now being compared to a conventional 30-fraction regime in a phase III trial [11]. We are currently investigating the extent to which hypofractionation can be safely achieved (ACTRN12619001186145) [12]. To accomplish this requires the most accurate image guidance possible to account for the daily position of organs-at-risk.

One of the key dose-limiting organs-at-risk when irradiating locally-advanced NSCLC is the oesophagus. Conventional fractionations with concurrent chemotherapy can result in $\geq$ Gr. 3 oesophagitis rates of $5-18 \%$ [13, 14], whilst hypofractionated regimes pose a greater risk of severe and even fatal reaction [10, 15]. The day-to-day position of the oesophagus can be inconsistent and is not fully accounted for during simulation and treatment planning [16-18]. Additionally, with standard cone-beam computed tomographic (CBCT) imaging, the visibility of the oesophagus is poor due its small size and similar radiographic density to the adjacent tissues. With a narrow therapeutic index, the oesophagus represents a significant obstacle in utilising SBRT for targets within the mediastinum $[19,20]$.

We have previously investigated the use of oral contrast with thoracic CBCT and compared $50 \mathrm{ml}$ each of Gastrografin and Barium Sulfate [21]. Barium allowed better visualisation of the oesophagus on $\mathrm{CBCT}$, however its density and volume led to artifacts which potentially impaired IGRT for other structures including the tumour itself. Qiu et al., using an even larger volume of barium, quantified interfractional oesophageal movement in relation to bony anatomy, and found this to be as high as $36 \mathrm{~mm} \mathrm{[18].} \mathrm{The} \mathrm{aim} \mathrm{of} \mathrm{this} \mathrm{work} \mathrm{was} \mathrm{to} \mathrm{determine} \mathrm{the}$ optimal volume of barium required to maintain oesophageal visibility and minimise imaging artifacts. Additionally, we aimed to quantify the interfraction motion of the oesophagus relative to the tumour, an essential IGRT consideration in applying SBRT for locally-advanced NSCLC.

\section{Methods}

\section{Patients and imaging}

NSCLC patients were prospectively recruited into this institutional ethics approved study after providing informed written consent. Patients were included if they were being planned for a radical course of treatment for disease that extended into the mediastinum and/or hilum in which the oesophagus was a primary organ at risk.

Undiluted barium sulfate (Liquibar $62.5 \% \mathrm{w} / \mathrm{w}, \mathrm{MCI}$ Forrest) was administered just prior to acquisition of the patient's planning $4 \mathrm{DCT}$ and prior to patient setup on the treatment couch for every alternate weekly CBCT. 4DCT scans were acquired in free breathing with contouring and dosimetry performed on the reconstructed average intensity projection dataset. The barium was contoured in the treatment planning system and the density was overridden and assigned a Hounsfield Unit of zero. This was to account for the institution's treatment planning algorithm's inability to accurately account for very high densities (Analytical Anisotropic Algorithm, Eclipse, v13.6, Varian Medical Systems, Palo Alto, CA). The variation in dose was estimated to be insignificant, due to the small size of the oesophagus compared to the treatment area and the multi-field, intensity modulated nature of the treatment plans. CBCTs were acquired in free breathing using thorax-specific protocols either in full rotation $\left(360^{\circ}\right.$, half-fan mode, $45 \mathrm{~cm}$ field-of-view) or half rotation "spotlight" mode $\left(200^{\circ}\right.$, full-fan mode $25 \mathrm{~cm}$ field-of-view) and reconstructed to $2-2.5 \mathrm{~mm}$ slice thickness (Varian Clinac iX; $110 \mathrm{kVp}$ or Varian TrueBeam; $125 \mathrm{kVp}$ ). Patients were sequentially assigned to 4 barium dose levels: $25 \mathrm{~mL}, 15 \mathrm{~mL}, 10 \mathrm{~mL}$ and $5 \mathrm{~mL}$. Barium was administered orally immediately prior to lying on the treatment couch. The time between barium administration and $\mathrm{CBCT}$ acquisition was recorded.

\section{Oesophageal visibility}

We assumed visualisation of the oesophagus during IGRT would correlate directly and objectively with manually generated contours. Hence, we utilised interobserver contouring reproducibility as a surrogate for oesophageal visualisation. The oesophagus was contoured on each planning CT and CBCT by six observers consisting of three radiation oncologists and three SBRTtrained radiation therapists. They were instructed to contour the oesophagus from $1 \mathrm{~cm}$ above to $1 \mathrm{~cm}$ below the planning target volume (PTV). Window levelling was up to the discretion of the observer as they would have during IGRT. All contouring was performed in the Aria Contouring Workspace (v13.6, Varian Medical Systems, Palo Alto, CA) with $0.1 \mathrm{~cm}$ resolution. The datasets were then transferred to the Computational Environment for Radiotherapy Research (CERR) software program [22] where a consensus contour was generated from the six observer contours using the simultaneous truth and performance level estimation (STAPLE) method [23]. This contour was then considered the ground truth for each dataset. Each observer contour was then compared to the consensus contour to determine the variation between them (see study flowchart in Fig. 1). How well the observers were able to visualise the oesophagus was thereby determined by how similar their contours were to the consensus contour. This was carried out separately using data from CBCTs with and without barium and the comparison was considered the improvement in visibility with barium. 


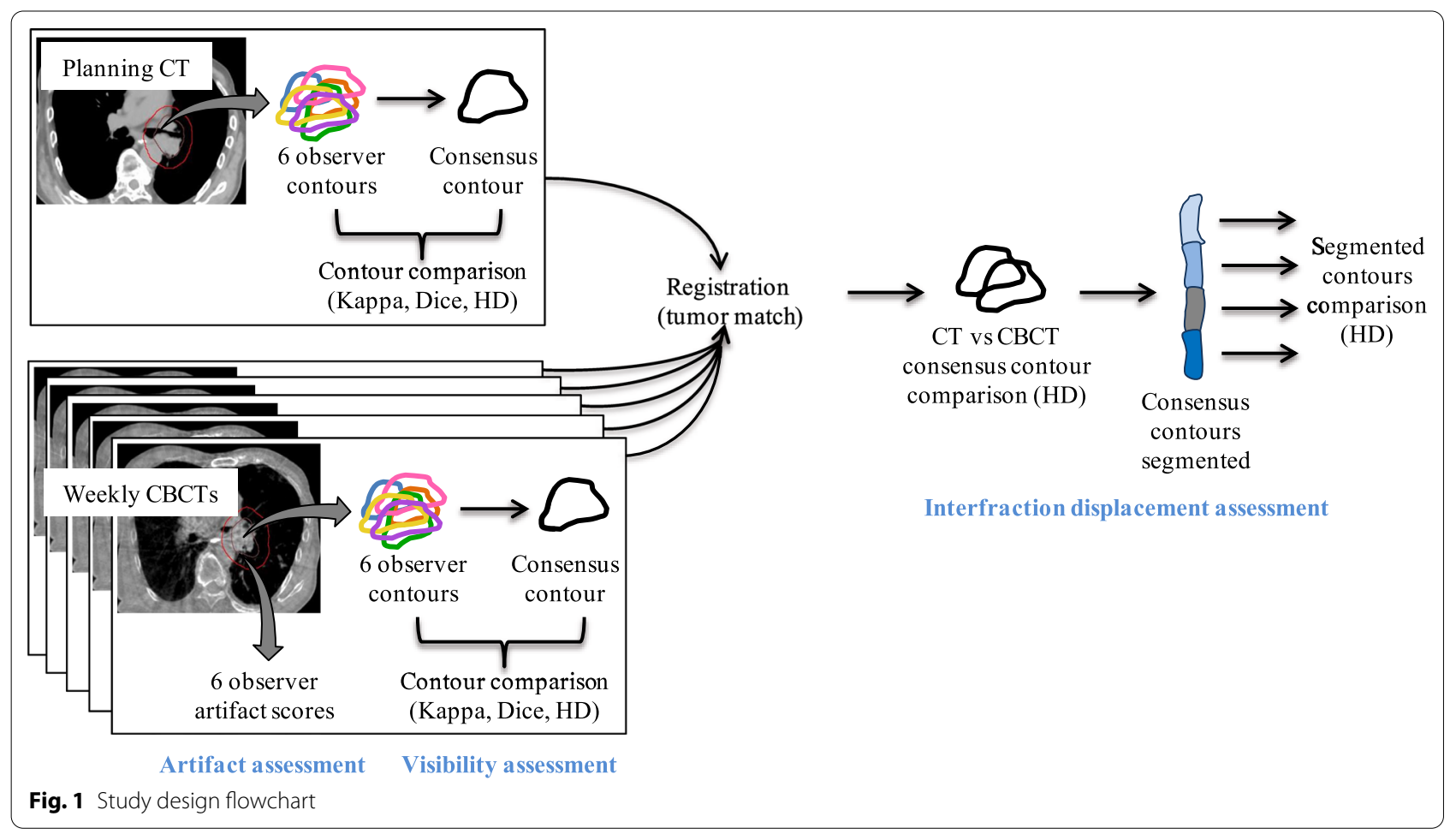

\section{Interfraction oesophageal motion}

Once a consensus contour was created for each planning CT and CBCT, the datasets were reimported to Aria. In the Image Registration Workspace each barium CBCT was rigidly registered with the planning $\mathrm{CT}$ based off a soft tissue tumour match and the consensus contours were then compared. We considered this difference to be due to variation in the oesophageal position over the course of treatment in relation to the tumour. Each consensus contour was then segmented into four sections to assess for trends in oesophageal motion based on anatomical levels. These sections were cervical (cricoid cartilage to sternal notch), upper (sternal notch to carina), middle (carina to midpoint between carina and gastroesophageal junction (GEJ)) and lower (midpoint between carina and GEJ to GEJ).

\section{Contour analysis}

The contours were compared using three metrics standardly used to compare differences in contours [24]; a kappa statistic [25], a dice coefficient [26] and a Hausdorff Distance (HD)(millimetres) [27]. A summary of these metrics and their use in this study can be found in Additional file 1: Table S1. The kappa statistic was calculated in CERR, whilst the dice coefficient and HD were calculated in the SlicerRT extension of the 3D Slicer software program [28]. The interobserver contouring reproducibility assessments utilised all three metrics. The interfraction motion assessments used the HD only as the primary objective of this analysis was to understand the gross difference in the oesophagus position in the context of daily online matching and its potential dosimetric implications.

\section{Image artifact assessment}

A qualitative assessment of contrast-induced image artifact was carried out. Each of the 6 observers scored the impact of any artifact on their ability to identify the gross tumour volume (GTV), trachea, main bronchi and central vessels on each CBCT dataset. Scores were defined as: $1=$ no impact, $2=$ some impact, but IGRT still achievable, $3=$ Significant impact, unable to identify sufficiently for IGRT purposes. Observers were not blinded to contrast volumes, however assessments were done throughout the study period over many months making bias less likely.

\section{Statistical analysis}

The median kappa, dice coefficient and HD were compared between CBCTs with and without contrast using a Mann-Whitney $U$ test. Differences between contrast dose levels were compared using a Kruskall Wallis test with post hoc Dunn-Bonferroni pairwise comparisons. Image artifact scores were reported using descriptive statistics. Correlation between the improvement of interobserver contouring reproducibility metrics gained with 
barium and the non-barium metrics, contouring reproducibility and time from contrast administration, and interfraction oesophageal displacement and PTV volume or overlap volume of PTV with oesophagus were carried out using a Pearson's correlation. Differences in interfraction oesophageal displacement between categorical variables (primary tumour laterality, thoracic nodal station involvement, involvement of mediastinal nodes, oesophagus segment) were compared using a Mann-Whitney $U$ test. Statistical analysis was carried out in SPSS Statistics (IBM Corp. v26.0. Armonk, NY). Significance for all tests was deemed as $p<0.05$.

\section{Results}

\section{Patient characteristics}

Of the 20 patients recruited, two patients chose to withdraw from the study-one could not tolerate barium at simulation and the other felt unwell in the days after simulation and attributed this to barium. Patient disease and treatment details are summarised in Table 1. Most patients had right-sided $(\mathrm{n}=10)$, stage III disease $(\mathrm{IIIA}=8, \mathrm{IIIB}=5, \mathrm{IIIC}=1)$ and received a median of 30 fractions (range: 15-30). Two patients had small amounts of pleural effusion, one patient had atelectasis and one patient had organising pneumonia at the start of treatment. The median PTV volume was $374.9 \mathrm{cc}$ and contoured oesophagus length was $12 \mathrm{~cm} .17$ planning CTs and $90 \mathrm{CBCTs}$ (44 with contrast, 46 without) were available for contouring and a total of 642 oesophagus structures were contoured by the 6 observers. One patient did not receive barium prior to their planning $\mathrm{CT}$ and so that dataset was not used for analysis.

\section{Oesophageal visibility}

Table 2 summarises the median and interquartile range of each metric for the planning CT datasets and the $\mathrm{CBCT}$ datasets. Barium significantly improved interobserver contouring reproducibility on CBCTs across all three metrics compared to $\mathrm{CBCTs}$ without barium. A significant difference between barium dose levels was found for the kappa statistic and dice coefficient, with the pairwise comparison showing a significant benefit only in the $10 \mathrm{~mL}$ level. There was no difference in the HD between dose levels. These results are also demonstrated in the boxplots of each metric grouped by dose level in Fig. 2.

The amount of improvement in interobserver contouring reproducibility gained with barium per patient correlated with the non-contrast CBCT metrics. In summary, the poorer the contouring reproducibility on the noncontrast $\mathrm{CBCT}$ the greater the improvement the barium provided. The metric improvements gained by each patient are displayed in Fig. 3. For the Dice coefficient and Kappa statistic there was a very strong $(\mathrm{r}=-0.909$, $p<0.001)$ and strong $(\mathrm{r}=-0.807, p<0.001)$ negative correlation respectively between the improvement gained and the baseline reading for either metric, whilst the HD demonstrated a very strong positive correlation $(\mathrm{r}=0.921, p<0.001)$.

Two patients did not have time measurements for analysis. Of those that did the median time between contrast administration and $\mathrm{CBCT}$ acquisition was $6 \mathrm{~min}$ (2-16 min). All CBCTs except two were acquired within 10 min of contrast consumption, with the largest time of 16 min occurring due to a delay caused by equipment breakdown. No correlation between the contour comparison metrics and time was found (Kappa $r=0.168$, $p=0.306$, Dice $\mathrm{r}=0.268, p=0.099, \mathrm{HD} \mathrm{r}=-0.024$, $p=0.883$ ). The median kappa statistic and HD were significantly higher in datasets acquired using the half rotation "spotlight" protocol compared to the full rotation protocol (Kappa 0.790 vs. $0.750, p=0.018$, Dice 0.884 vs. $0.860, p=0.072$ and HD 1.8 vs. $2.2 \mathrm{~mm}, p=0.009$ ).

\section{Contrast-induced image artifact}

The scoring of detrimental artifact was variable across the dose levels. Of the 1,056 total scores recorded $4 \%$, $10 \%, 6 \%$ and $8 \%$ were scores of 3 in the $25 \mathrm{~mL}, 15 \mathrm{~mL}$, $10 \mathrm{~mL}$ and $5 \mathrm{~mL}$ dose levels respectively. This compares to $12 \%$ found in our previous study using $50 \mathrm{~mL}$ of barium [21]. Of the 71 scores of 3 recorded, the majority were for central vessels (39\%), followed by GTV (30\%), Bronchi (27\%) and Trachea (4\%). A score of 3 was more common in some patients than in others, with $39 \%$ having no scores of 3 recorded and $61 \%$ having no scores of 3 for the GTV. A moderate positive correlation was found between the average GTV artifact score and the percentage of the PTV overlapping with the oesophagus $(\mathrm{r}=0.527, p=0.025)$. Of the 7 patients with a score of 3 for the GTV, 6 of these showed marked improvements in each of their 3 contouring reproducibility metrics. The perception of the artifact also appeared to be observer dependent. $100 \%, 46 \%, 64 \%$ and $63 \%$ of scores of 3 came from the same observer in the $25 \mathrm{~mL}, 15 \mathrm{~mL}, 10 \mathrm{~mL}$ and $5 \mathrm{~mL}$ dose levels, while another observer did not score any region of interest on any dataset with a 3 .

\section{Interfraction oesophageal displacement}

Comparing the consensus contours between the barium CBCTs and the planning CTs, $69 \%$ of patients had a reduction in oesophagus volume by an average of $4.2 \mathrm{cc}$ in the first 2-3 weeks of treatment and half of those saw a further reduction in week 4 . Of those patients who had a barium CBCT in weeks 5 or $6,63 \%$ had a reduced volume compared to the planning $\mathrm{CT}$. 


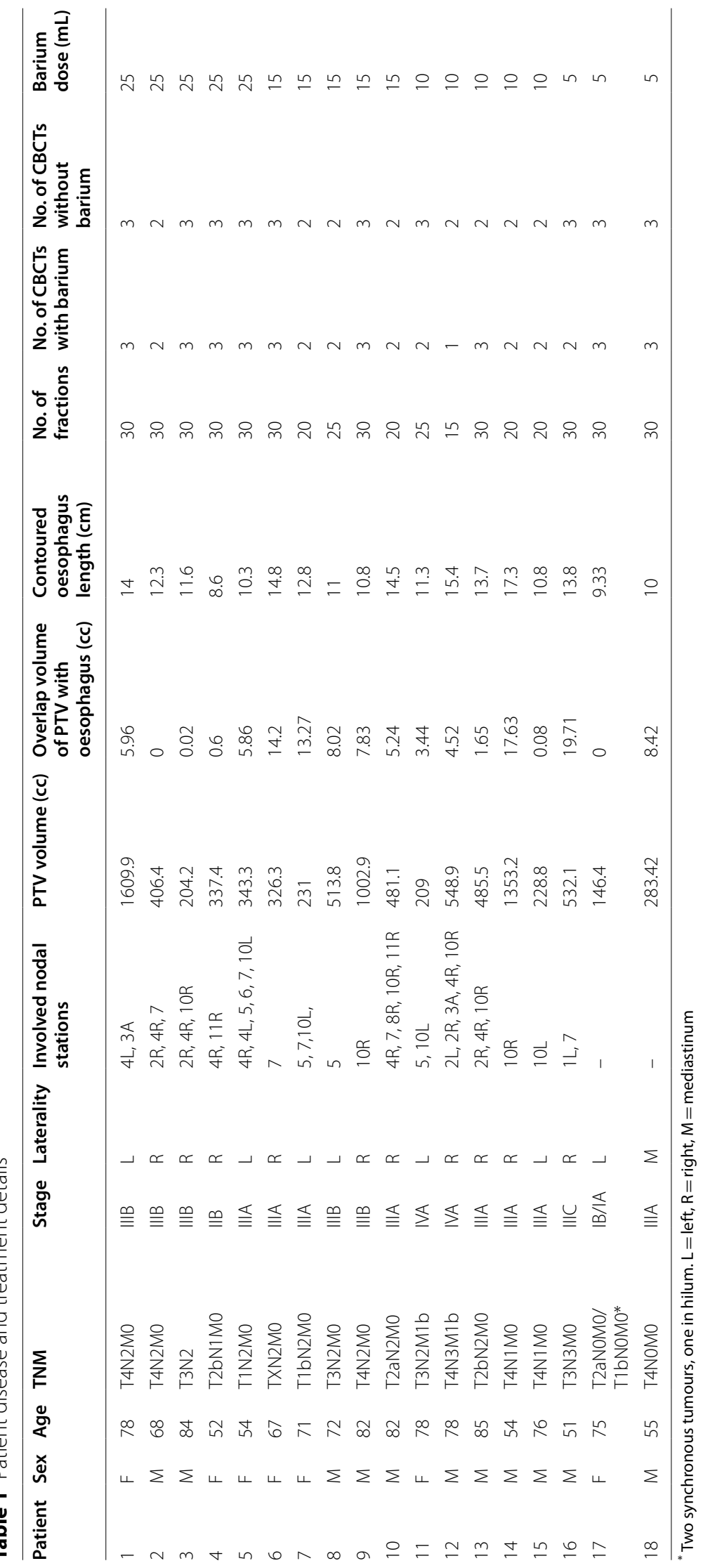


Table 2 Summary of the median [IQR] Kappa, Dice and HD on the planning CTs and CBCTs

\begin{tabular}{|c|c|c|c|c|c|c|c|c|c|}
\hline & \multirow{2}{*}{$\begin{array}{l}\text { Planning CTs } \\
\text { (with Barium) } \\
(\mathrm{n}=17)\end{array}$} & \multirow{2}{*}{$\begin{array}{l}\text { CBCTs without } \\
\text { Barium } \\
(n=46)\end{array}$} & \multirow{2}{*}{$\begin{array}{l}\text { CBCTs with } \\
\text { Barium } \\
(n=44)\end{array}$} & \multirow[t]{2}{*}{$p$-value* } & \multicolumn{5}{|c|}{ CBCTs with Barium } \\
\hline & & & & & $25 \mathrm{~mL}(\mathrm{n}=14)$ & $15 \mathrm{~mL}(\mathrm{n}=12)$ & $10 \mathrm{~mL}(\mathrm{n}=10)$ & $5 \mathrm{~mL}(\mathrm{n}=8)$ & $p$-value * \\
\hline Kappa & $0.851[0.037]$ & $0.607[0.162]$ & $0.772[0.096]$ & $<0.001$ & $0.732[0.061]$ & $0.766[0.132]$ & $0.814[0.043]$ & $0.775[0.112]$ & 0.008 \\
\hline Dice & $0.916[0.039]$ & $0.791[0.161]$ & $0.879[0.071]$ & $<0.001$ & $0.859[0.085]$ & $0.866[0.077]$ & $0.895[0.030]$ & $0.883[0.102]$ & $<0.001$ \\
\hline \multirow[t]{9}{*}{$\mathrm{HD}(\mathrm{mm})$} & $1.3[0.5]$ & $3.9[2.6]$ & $2.0[1.0]$ & $<0.001$ & $2.1[1.1]$ & 2.0 [1.3] & $1.9[0.7]$ & 1.9 [1.2] & 0.220 \\
\hline & & & & & \multicolumn{5}{|c|}{$p$-values for Dunn-Bonferroni pairwise comparisons* } \\
\hline & & & & & \multicolumn{2}{|l|}{ Kappa } & \multicolumn{2}{|l|}{ Dice } & HD \\
\hline & & & $25-15 \mathrm{~mL}$ & & \multicolumn{2}{|l|}{1.000} & \multicolumn{2}{|l|}{1.000} & 1.000 \\
\hline & & & $25-10 \mathrm{~mL}$ & & \multicolumn{2}{|l|}{0.006} & \multicolumn{2}{|l|}{$<0.001$} & 1.000 \\
\hline & & & $25-5 \mathrm{~mL}$ & & \multicolumn{2}{|l|}{1.000} & \multicolumn{2}{|l|}{1.000} & 1.000 \\
\hline & & & $15-10 \mathrm{~mL}$ & & \multicolumn{2}{|l|}{0.051} & \multicolumn{2}{|l|}{$<0.001$} & 1.000 \\
\hline & & & $15-5 \mathrm{~mL}$ & & \multicolumn{2}{|l|}{1.000} & \multicolumn{2}{|l|}{1.000} & 1.000 \\
\hline & & & $10-5 \mathrm{~mL}$ & & \multicolumn{2}{|l|}{0.249} & \multicolumn{2}{|l|}{0.036} & 1.000 \\
\hline
\end{tabular}

* $P$-values from the Mann-Whitney test (CBCTs—Barium vs. no Barium) and Kruskall-Wallis test (between Barium dose levels) with post hoc analysis

After registering each barium $\mathrm{CBCT}$ with the planning CT, the median HD between the consensus contours was $3.9 \mathrm{~mm}$ (range $2.2-25.5 \mathrm{~mm}$ ). Figure 4 shows the variation of these HD measurements (planning CT vs. CBCTs) for each patient over the course of treatment, noting the HD provides magnitude and not direction of displacement. $76 \%$ of the variation was within $5 \mathrm{~mm}$ suggesting a systematic difference, potentially due to the use of CBCT as opposed to the planning CT. Despite this trend larger, more random, deviations were observed for some patients and fractions. $14 \%$ and $5 \%$ of the measurements were over $10 \mathrm{~mm}$ and $15 \mathrm{~mm}$ respectively. There was no significant difference in median HD between the four anatomical segments of the oesophagus (cervical $3.0 \mathrm{~mm}$, upper $3.7 \mathrm{~mm}$, middle $3.2 \mathrm{~mm}$, lower $3.6 \mathrm{~mm}$, $p=0.135$ ). No correlation was found between the HD and primary tumour laterality (right $3.9 \mathrm{~mm}$, left $3.4 \mathrm{~mm}$, $p=0.862$ ) or the volume of PTV overlapping the oesophagus $(\mathrm{r}=0.050, p=0.751)$. A weak correlation was seen between oesophagus displacement and PTV volume $(r=0.392, p=0.010)$. There was no significant difference between displacement and any of the involved individual lymph node stations. A significantly smaller HD was recorded in patients with mediastinal lymph node involvement than in those without $(3.4 \mathrm{~mm}$ vs. $5 \mathrm{~mm}$, $p=0.008)$.

\section{Discussion}

We have demonstrated that a small amount of barium is sufficient in improving the interobserver contouring reproducibility of the oesophagus on $\mathrm{CBCT}$ imaging with no improvement with increasing dose. Barium improved the contouring reproducibility metrics from baseline for each patient. The amount of improvement the contrast provided varied between patients and was less dose dependent than it was patient dependent. Improvement correlated with the baseline measurement, indicating the poorer the interobserver contouring reproducibility without contrast, the greater the improvement the contrast provided. From these results we infer that the improved precision in observer contouring corresponds to improved oesophageal visibility due to barium administration. The only dose level that showed a significant improvement over the others was $10 \mathrm{~mL}$, although this was found in the Kappa and Dice metrics and not the HD. Previous reports have shown that the Dice and HD do not correlate and instead complement one another [29], highlighting the importance of using a combination of metrics in studies like this [24].

There is growing evidence to show that hypofractionated, SBRT-like treatments may play a significant role in patients with locally-advanced NSCLC who are ineligible for concurrent chemoradiotherapy [8-11,30]. These regimes can cause severe toxicity to central organs like the oesophagus. The oesophagus is mobile and its position is influenced by both normal physiological functions (peristalsis, swallowing, respiration, cardiac rhythm) and tumour-related changes (tumour regression/progression, atelectasis, plural effusion) $[16,31]$. Its position cannot be inferred by bony landmarks, nor assumed to be consistent over a course of radiotherapy spanning weeks, hence monitoring its daily position is paramount. We found the median interfraction oesophagus HD was $4 \mathrm{~mm}$ compared to the planning CT with $14 \%$ measuring over $10 \mathrm{~mm}$. The magnitude of displacement could not be predicted by anatomical level, primary tumour laterality, overlap of the PTV with the oesophagus, nor by involvement of specific nodal stations. No patients in this study 

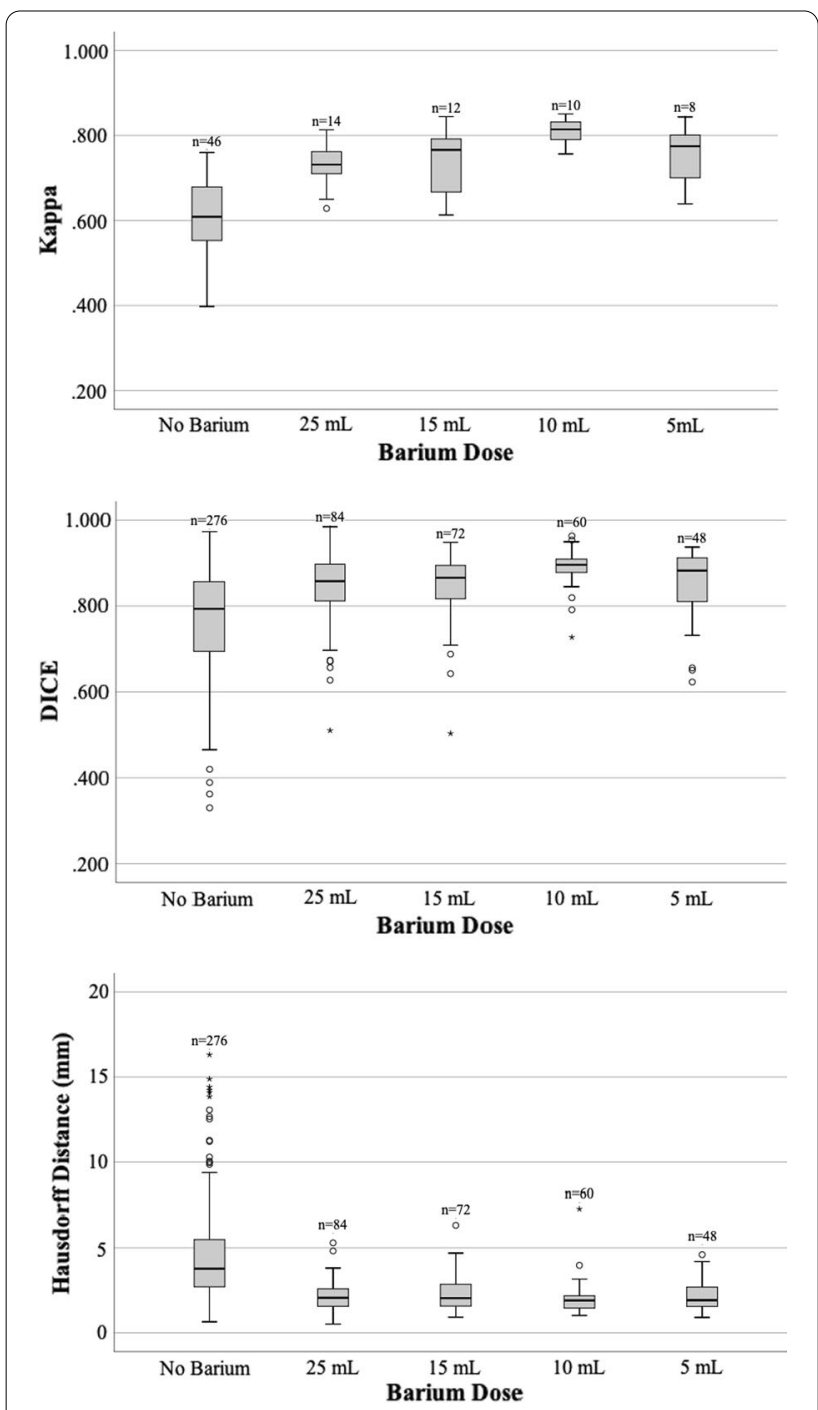

Fig. 2 Boxplots summarising the Kappa, Dice and Hausdorff Distance metrics per barium dose level. ${ }^{*}$ A higher Kappa and Dice score and a smaller Hausdorff Distance represents better reproducibility between observer contours. Outlier at HD=29 mm in No Barium group not depicted as out of scale had significant tumour changes that required re-simulation, however reports have shown this can occur in $20 \%$ of locally-advanced lung cancer patients with significant risk to mediastinal structures if not actioned upon [32]. Figure 5 demonstrates a patient from this study with extreme variation in oesophageal position from as early as week 2 through to week 6 . It could be argued that the introduction of an oral contrast agent to the oesophageal lumen may induce oesophageal motion through peristalsis. Given the small volume of barium used, the rapid nature of oesophageal transit times (seconds) [33] and the timeframe from administration to CBCT (minutes) this is unlikely to be a significant consideration. We also found the oesophageal volume decreased over the treatment course in most patients. We speculate that this is due to treatment related oesophagitis, causing constriction and luminal narrowing [34]. This then results in less barium accumulating in the lumen and as the external boundary of the lumen can be difficult to see on CBCT, the contoured volumes have shrunk.

Qiu et al. [18] have also recently reported on the use of barium with pre-treatment $\mathrm{CBCTs}$ for this purpose. They utilised $30 \mathrm{~mL}$ of barium before and a further $30 \mathrm{~mL}$ during $\mathrm{CBCT}$ acquisition and found oesophageal motion was greater in the left-right direction than the anterior-posterior direction, more prominent in the middle oesophagus and in patients with right sided disease or with bulky mediastinal lymph nodes. However, these positional variations were reported with reference to the bony landmarks. It is well understood that thoracic tumours move independent of bones $[35,36]$ and so in the context of hypofractionated SBRT treatments online soft tissue match to tumour is preferred [37]. We thus sought in this study to understand the relationship of the oesophageal position in relation to the tumour position over the course of treatment to help guide dosimetric decisions during treatment planning. The addition of barium to the planning and treatment of these patients can also help guide and verify the position of high dose
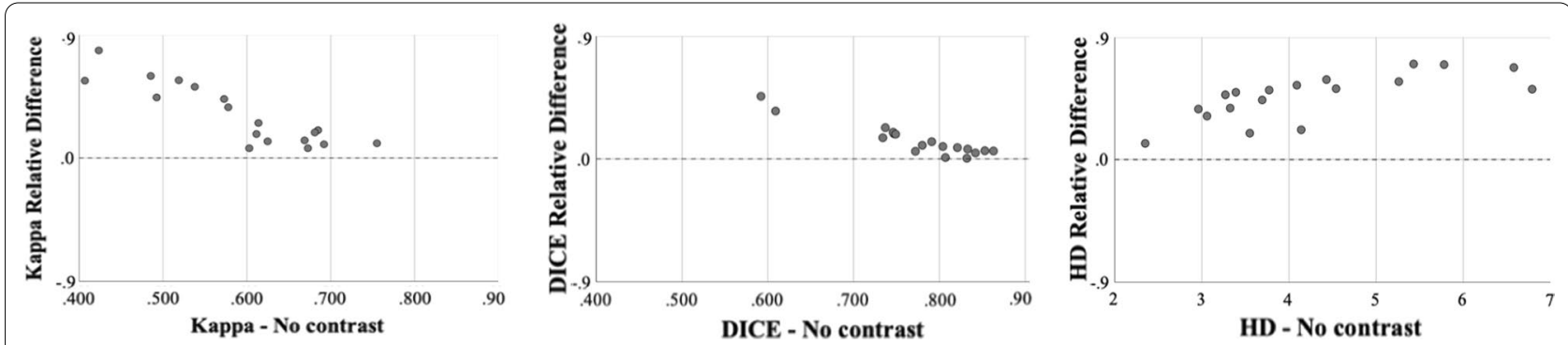

Fig. 3 Relative difference in median Kappa, Dice and HD on barium CBCTs compared to non-contrast CBCTs. A positive difference refers to an improvement in the metric gained by barium use. Each dot represents a patient 


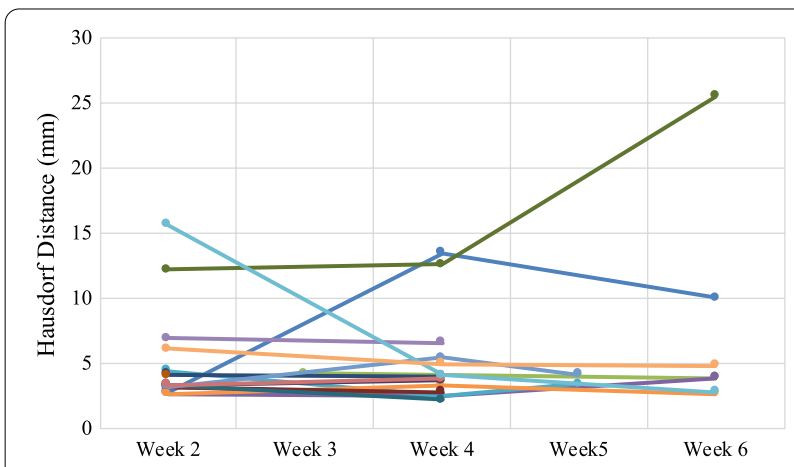

Fig. 4 Gross oesophageal displacement between planning CT and barium CBCT over the treatment course. Each line/colour represents a patient

gradients, for example when utilising a contralateral oesophageal-sparing technique [38, 39].

The consequence of barium-induced image artifact on CBCT has not been investigated until now. Artifact perception was not dose dependent as we had hypothesized, but instead influenced by individual patient and tumour anatomy. Proximity of organs/targets to the oesophagus had a greater impact than barium dose. Artifact influenced visibility of the central vessels more than other organs, which is acceptable given they are not as dose-restricting and their position is consistent. It remains of concern that some observers perceived the artifact to impede their ability to visualise the GTV. The more the PTV overlapped with the oesophagus, the greater the chance the barium influenced one's ability to clearly see the GTV on CBCT. However, in nearly all of these patients the oesophageal visibility was greatly improved by the barium, so a costbenefit question arises in some patients between oesophageal and GTV visibility. Furthermore, the perception of image artifact is subjective and varies significantly between observers. In practice this subjectivity may be reduced because multiple staff are generally involved with online pre-treatment $\mathrm{CBCT}$ evaluation.

We found that in order to capture $95 \%$ of the oesophageal positional variations during treatment planning a planning risk volume (PRV) of $15 \mathrm{~mm}$ would be required. Qiu et al. [18] reported smaller 95\% margins between 2.8 and $10.3 \mathrm{~mm}$ depending on location and direction, whilst Cohen et al. reported $8-12 \mathrm{~mm}$ although theirs
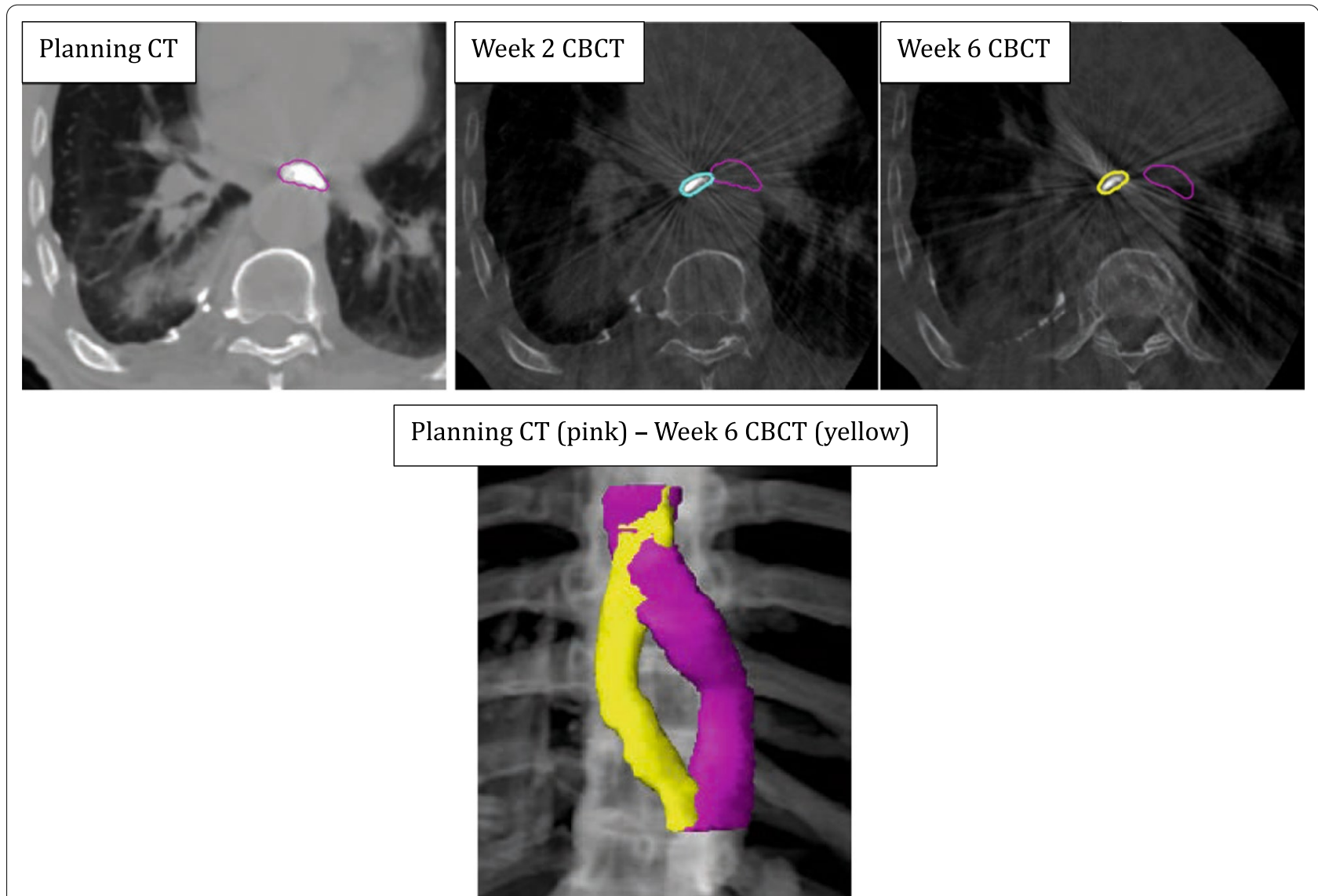

Fig. 5 Variation in oesophageal position from planning CT to week 2 CBCT and week 6 CBCT of one patient 
reported from CBCTs of oesophageal cancers [40]. The limits of SBRT for locally-advanced NSCLC are not yet known and we are investigating this in a phase 1 dose escalation trial [12]. To incorporate a $15 \mathrm{~mm}$ PRV around the oesophagus will be impractical for most node positive NSCLC undergoing SBRT and a large PRV may jeopardise tumour control. Given a proportion of the shifts appeared random in nature (Fig. 4) the best solution may be online adaptive treatment strategies where the precise oesophageal position of the day is determined and the patient simply not treated if there is a significant shift into the high dose region. Additionally, with accurate visualisation and determination that a shift is more systematic, replanning or adaptive changes can be initiated.

This study has a number of limitations. The patient numbers are small and the results highlight the great inhomogeneity that this disease cohort presents. As the sample size per barium dose group was small, eliminating the influence of patient or disease variables on the results per dose group was impractical. Similarly, the number of treatment fractions, weekly CBCTs and patient numbers differed between groups, which may have influenced the results. Our finding of improved interobserver contouring reproducibility with barium demonstrates improved precision of oesophageal localisation. It does not necessarily, however demonstrate improved accuracy of oesophageal position. Considering the image quality of a linac CBCT (and not an MRI-linac for example) the true position of the oesophagus cannot be accurately determined. As with all radiotherapy studies drawing conclusion from human-generated contours, the resultant ground truth is dependent on the number and expertise of the observers. We intentionally chose both radiation oncologists and SBRT-trained therapists to participate as this reflects the true allocation of responsibility in the clinic. The study is further strengthened by the use of 6 observers to contour and assess image-artifact on each dataset, reducing the influence of investigator bias. Finally, in this study CBCTs were acquired weekly as per department protocol for conventionally fractionated radical treatment. Our results could have benefited had daily CBCTs been used. This study could be repeated in the future in patients receiving a hypofractionated course when daily CBCTs would be standard.

\section{Conclusions}

Small doses of barium delivered conveniently prior to patient setup greatly improve the interobserver contouring reproducibility of the oesophagus on CBCT and do not significantly degrade the image quality. Barium has helped show that the position of the oesophagus in relation to the tumour is variable and unpredictable over a course of treatment, requiring careful dosimetric consideration, close monitoring and ideally adaptive treatment strategies.

\begin{abstract}
Abbreviations
4DCT: 4-Dimensional computed tomography; CBCT: Cone beam computed tomography; CERR: Computational environment for radiotherapy research; $C T$ : Computed tomography; GEJ: Gastroesophageal junction; GTV: Gross tumour volume; HD: Hausdorff Distance; IGRT: Image-guided radiotherapy; IQR: Interquartile range; LA: Locally-advanced; NSCLC: Non-small cell lung cancer; PRV: Planning risk volume; PTV: Planning target volume; SBRT: Stereotactic body radiotherapy; STAPLE: Simultaneous truth and performance level estimation; TNM: Tumour node metastasis.
\end{abstract}

\section{Supplementary Information}

The online version contains supplementary material available at https://doi. org/10.1186/s13014-021-01946-8.

Additional file 1. Contour analysis metrics and their use in this study.

\section{Acknowledgements}

Not applicable.

\section{Authors' contributions}

KW, VP, SS, JR, SD were responsible for the study design. KW, TTL and SM was responsible for the data collection. KW, SS, JR, SD, TTL and SM were responsible for the contouring. KW, VP and SS were responsible for the data analysis. $\mathrm{KW}$ was the main contributor for the writing of the manuscript. All authors read and approved the final manuscript.

Funding

No funding was received for the research reported herein

Availability of data and materials

The datasets used and/or analysed during the current study are available from the corresponding author on reasonable request.

\section{Declarations}

Ethics approval and consent to participate

Ethics approval was received from Alfred Health's Human Research and Ethics Committee. Project ID: 136-16. All participants in this study provided informed written consent prior to participation.

\section{Consent for publication}

Not applicable.

\section{Competing interests}

The authors declare that they have no competing interests.

\section{Author details}

${ }^{1}$ Alfred Health Radiation Oncology, The Alfred, 55 Commercial Road, Melbourne, VIC 3004, Australia. 'Department of Surgery, Central Clinical School, Monash University, Melbourne, VIC, Australia. ${ }^{3}$ Department of Medical Imaging and Radiation Sciences, Monash University, Clayton, VIC, Australia.

Received: 23 June 2021 Accepted: 3 November 2021

Published online: 14 November 2021

References

1. Adizie JB, Khakwani A, Beckett P, Navani N, West D, Woolhouse I, et al. Stage III non-small cell lung cancer management in England. Clin Oncol. 2019;31(10):688-96 
2. Driessen EJM, Bootsma GP, Hendriks LEL, van den Berkmortel FWPJ, Bogaarts BAHA, van Loon JGM, et al. Stage III non-small cell lung cancer in the elderly: patient characteristics predictive for tolerance and survival of chemoradiation in daily clinical practice. Radiother Oncol. 2016;121(1):26-31.

3. Coate LE, Massey C, Hope A, Sacher A, Barrett K, Pierre A, et al. Treatment of the elderly when cure is the goal: the influence of age on treatment selection and efficacy for stage III non-small cell lung cancer. J Thorac Oncol. 2011:6(3):537-44.

4. Wang S, Wong ML, Hamilton N, Davoren JB, Jahan TM, Walter LC. Impact of age and comorbidity on non-small-cell lung cancer treatment in older veterans. J Clin Oncol. 2012;30(13):1447-55.

5. Haasbeek CJ, Palma D, Visser O, Lagerwaard FJ, Slotman B, Senan S. Early-stage lung cancer in elderly patients: a population-based study of changes in treatment patterns and survival in the Netherlands. Ann Oncol. 2012;23(10):2743-7.

6. Haque W, Szeja S, Tann A, Kalra S, Teh BS. Changes in treatment patterns and overall survival in patients with early-stage non-small cell lung cancer in the United States after the incorporation of stereotactic ablative radiation therapy: a population-based analysis. Am J Clin Oncol. 2018:41(3):259-66.

7. Dalwadi SM, Szeja SS, Bernicker EH, Butler EB, Teh BS, Farach AM. Practice patterns and outcomes in elderly stage I non-small-cell lung cancer: a 2004 to 2012 SEER analysis. Clin Lung Cancer. 2018;19(2):e269-76.

8. Westover KD, Loo BW Jr, Gerber DE, lyengar P, Choy H, Diehn M, et al. Precision hypofractionated radiation therapy in poor performing patients with non-small cell lung cancer: phase 1 dose escalation trial. Int J Radiat Oncol Biol Phys. 2015;93(1):72-81.

9. Pollom EL, Qian Y, Durkee BY, von Eyben R, Maxim PG, Shultz DB, et al. Hypofractionated intensity-modulated radiotherapy for patients with non-small-cell lung cancer. Clin Lung Cancer. 2016;17(6):588-94.

10. Kong C, Zhu X, Shi M, Wang L, Chen C, Tao H, et al. Survival and toxicity of hypofractionated intensity-modulated radiotherapy in 4-Gy fractions for unresectable stage III non-small-cell lung cancer. Int J Radiat Oncol Biol Phys. 2020;107(4):710-9.

11. Iyengar P, Westover KD, Court LE, Patel MK, Shivnani AT, Saunders MW, et al. A phase III randomized study of image guided conventional ( 60 $\mathrm{Gy} / 30 \mathrm{fx}$ ) versus accelerated, hypofractionated (60 Gy/15 fx) radiation for poor performance status stage II and III NSCLC patients: an interim analysis. Int J Radiat Oncol Biol Phys. 2016;96(2):E451.

12. Senthi S. Stereotactic ablative radiotherapy for locally-adavanced nonsmall cell lung cancer (ACTRN12619001186145): Australian New Zealand Clinical Trials Registry; 2020 [Available from: https://www.anzctr.org.au/ Trial/Registration/TrialReview.aspx?id=377558\&isReview=true.

13. Bradley JD, Hu C, Komaki RR, Masters GA, Blumenschein GR, Schild SE, et al. Long-term results of NRG oncology RTOG 0617: standard- versus high-dose chemoradiotherapy with or without cetuximab for unresectable stage III non-small-cell lung cancer. J Clin Oncol. 2020;38(7):706-14.

14. Palma DA, Senan S, Oberije C, Belderbos J, de Dios NR, Bradley JD, et al. Predicting esophagitis after chemoradiation therapy for non-small cell lung cancer: an individual patient data meta-analysis. Int J Radiat Oncol Biol Phys. 2013;87(4):690-6.

15. van Diessen J, De Ruysscher D, Sonke J-J, Damen E, Sikorska K, Reymen $B$, et al. The acute and late toxicity results of a randomized phase II doseescalation trial in non-small cell lung cancer (PET-boost trial). Radiother Oncol. 2019;131:166-73.

16. Dieleman EMT, Senan S, Vincent A, Lagerwaard FJ, Slotman BJ, van Sörnsen de Koste JR. Four-dimensional computed tomographic analysis of esophageal mobility during normal respiration. Int J Radiat Oncol Biol Phys. 2007; 67(3):775-80.

17. Yamashita H, Haga A, Hayakawa Y, Okuma K, Yoda K, Okano Y, et al. Patient setup error and day-to day esophageal motion error analyzed by cone-beam computed tomography in radiation therapy. Acta Oncol. 2010;49:485-90.

18. Qiu B, Lu SP, Wang B, Shao JH, Zhang YT, Yun Qiang M, et al. Quantifying the interfractional motion of esophagus using daily cone beam computed tomography with oral contrast during radiotherapy for locally advanced non-small cell lung cancer. Pract Radiat Oncol. 2020:10(5):E339-47.

19. Abelson JA, Murphy JD, Loo BW Jr, Chang DT, Daly ME, Wiegner EA, et al. Esophageal tolerance to high-dose stereotactic ablative radiotherapy. Dis Esophagus. 2012;25(7):623-9.
20. Duijm M, van der Voort van Zyp NC, van de Vaart P, Hoop EO-D, Mast ME, Hoogeman MS, et al. Predicting high-grade esophagus toxicity after treating central lung tumors with stereotactic radiotherapy using a Normal Tissue Complication Probability model. Int J Radiat Oncol Biol Phys. 2020; 106(1):73-81.

21. Woodford K, Panettieri V, Ruben JD, Davis S, Sim E, Tran Le T, et al. Contrast enhanced oesophageal avoidance for stereotactic body radiotherapy: Barium vs. Gastrografin. Tech Innov Patient Support Radiat Oncol. 2019;12:16-22.

22. Deasy JO, Blanco Al, Clark VH. CERR: a computational environment for radiotherapy research. Med Phys. 2003;30(5):979-85.

23. Warfield SK, Zou KH, Wells WM. Simultaneous truth and performance level estimation (STAPLE): an algorithm for the validation of image segmentation. IEEE Trans Med Imaging. 2004;23(7):903-21.

24. Fotina I, Lutgendorf-Caucig C, Stock M, Potter R, Georg D. Critical discussion of evaluation parameters for inter-observer variability in target definition for radiation therapy. Strahlenther Onkol. 2012;188(2):160-7.

25. McHugh ML. Interrater reliability: the kappa statistic. Biochem Med. 2012;22(3):276-82.

26. Dice LR. Measures of the amount of ecologic association between species. Ecology. 1945;26(3):297-302.

27. Gardner SJ, Wen N, Kim J, Liu C, Pradhan D, Aref I, et al. Contouring variability of human- and deformable-generated contours in radiotherapy for prostate cancer. Phys Med Biol. 2015;60(11):4429-47.

28. Pinter C, Lasso A, Wang A, Jaffray D, Fichtinger G. SlicerRT_radiation therapy research toolkit for 3D Slicer. Med Phys. 2012;39(10):6332-8.

29. Sharp G, Fritscher KD, Pekar V, Peroni M, Shusharina N, Veeraraghavan H, et al. Vision 20/20: Perspectives on automated image segmentation for radiotherapy. Med Phys. 2014;41(5):050902.

30. Tekatli H, Haasbeek N, Dahele M, De Haan P, Verbakel W, Bongers E, et al. Outcomes of hypofractionated high-dose radiotherapy in poor-risk patients with "ultracentral" non-small cell lung cancer. JThorac Oncol. 2016;1 17):1081-9.

31. Kwint M, Conijn S, Schaake E, Knegjens J, Rossi M, Remeijer P, et al. Intra thoracic anatomical changes in lung cancer patients during the course of radiotherapy. Radiother Oncol. 2014;113(3):392-7.

32. Appel S, Bar J, Alezra D, Ben-Ayun M, Rabin-Alezra T, Honig N, et al. Image-guidance triggered adaptive replanning of radiation therapy for locally advanced lung cancer: an evaluation of cases requiring plan adaptation. Br J Radiol. 2019;93(1105):20190743.

33. Aly YA, Abdel-Aty H. Normal oesophageal transit time on digital radiography. Clin Radiol. 1999:54(8):545-9.

34. Benveniste MF, Gomez D, Carter BW, Betancourt Cuellar SL, Shroff GS, Benveniste APA, et al. Recognizing radiation therapy-related complications in the chest. Radiographics. 2019;39(2):344-66.

35. Hawkins M, Aitken A, Hansen V, McNair H, Tait D. Cone beam CT verification for oesophageal cancer-impact of volume selected for image registration. Acta Oncol. 2011;50(8):1183-90.

36. Knap MM, Hoffmann L, Nordsmark M, Vestergaard A. Daily cone-beam computed tomography used to determine tumour shrinkage and localisation in lung cancer patients. Acta Oncol. 2010;49(7):1077-84.

37. Purdie TG, Bissonnette JP, Franks K, Bezjak A, Payne D, Sie F, et al. Conebeam computed tomography for on-line image guidance of lung stereotactic radiotherapy: localization, verification, and intrafraction tumor position. Int J Radiat Oncol Biol Phys. 2007:68(1):243-52.

38. Al-Halabi H, Paetzold P, Sharp GC, Olsen C, Willers H. A contralateral Esophagus-Sparing technique to limit severe esophagitis associated with concurrent high-dose radiation and chemotherapy in patients with thoracic malignancies. Int J Radiat Oncol Biol Phys. 2015;92(4):803-10.

39. Kamran SC, Yeap BY, Ulysse CA, Cronin C, Bowes CL, Durgin B, et al. Assessment of a contralateral Esophagus-Sparing Technique in locally advanced lung cancer treated with high-dose chemoradiation: a phase 1 nonrandomized clinical trial. JAMA Oncol. 2021;7(6):910-4.

40. Cohen RJ, Paskalev K, Litwin S, Price RA Jr, Feigenberg SJ, Konski AA. Esophageal motion during radiotherapy: quantification and margin implications. Dis Esophagus. 2010;23(6):473-9.

\section{Publisher's Note}

Springer Nature remains neutral with regard to jurisdictional claims in published maps and institutional affiliations. 